\title{
Asthma in der Schwangerschaft
}

\section{Mädchen lassen ihre Mütter schnaufen}

Asthma ist eine Hauptursache für Schwangerschaftskomplikationen. Dabei kann sich die Krankheit während der Schwangerschaft und danach verändern. Kontrolle ist gefragt!

Unter Asthma leiden 8\% aller Schwangeren. Das erhöht ihr Risiko für Schwangerschaftserbrechen, Präeklampsie, vorzeitigen Blasensprung oder einen Kaiserschnitt. Dem werdenden Nachwuchs drohen eine erhöhte Mortalität, ein geringes Geburtsgewicht, eine zu frühe Geburt, kongenitale Missbildungen und Wachstumsretardierung. Eine gute therapeutische Kontrolle wirkt dem entgegen. Allerdings bleibt die Symptomatik nur bei einem Drittel der werdenden Mütter mit Asthma unverändert, bei vielen bessert sich das Asthma auch - oder verschlechtert sich. Deshalb empfiehlt PD Dr. Andrea Koch aus Köln bei schwangeren Asthmatikerinnen eine Asthmakontrolle alle ein bis drei Monate, wie sie beim Kongress der Deutschen Gesellschaft für Pneumologie und Beatmungsmedizin in Hannover berichtete.

\section{Jungen machen Asthma besser}

Die Asthmasymptomatik der Mutter ist überraschenderweise auch vom Geschlecht des Nachwuchses abhängig. Werdende Mütter mit Asthma leiden stärker unter Husten, Kurzatmigkeit und nächtlichem Erwachen, wenn sie ein Mädchen erwarteten. Bei einem Jungen im Bauch bessert sich das Asthma in dieser Zeit häufiger. Eine Wachstumsretardierung bei einem Asthma der Mutter fand sich auch eher bei Mädchen, nicht aber bei Jungen und auch nicht, wenn mit niedrig dosierten inhalativen Steroiden (ICS) behandelt worden war.

Bei leichtgradigem Asthma kann in der Schwangerschaft therapiert werden wie immer: Bei intermittierenden Sym- ptomen sollten bei Bedarf kurz wirksame inhalative $\beta_{2}$-Agonisten zur Verfügung stehen, bei leichtgradig persistierendem Asthma kommt dazu ein niedrig dosiertes ICS. Beim mittelgradigen Asthma kann eine mittlere Dosis (600-1200 $\mu \mathrm{g})$ ICS oder aber weiter eine niedrige Dosis $(<500 \mu \mathrm{g})$ ICS und ein inhalativ lang wirksamer $\beta_{2}$-Agonist (LABA) gegeben werden. Im Falle eines schwergradigen Asthmas muss das ICS hoch dosiert werden, wieder ergänzt um einen LABA.

Am sichersten im Hinblick auf das Fehlbildungsrisiko erscheint eine niedrige bis mittelhohe Dosis von inhalativem Budesonid, als kurz wirksamer $\beta_{2}$-Agonist wird Salbutamol, als LABA Salmeterol empfohlen. Alternativ können Theophyllin oder Montelukast eingesetzt werden, Letzteres auch als Add-on-Therapie. Der Einsatz oraler Steroide muss gegen ein erhöhtes Fehlbildungsrisiko (u. a. Lippen-Gaumen-Spalten) abgewogen werden.

$(F K)$

\section{Nicht alles ist Asthma Giemen nur an einem Ort? Verdächtig!}

Wenn es nur punktuell und nicht überall in der Lunge ungesund klingt, ist Vorsicht geboten. Denn mögliche Ursachen reichen dann von Polypen bis zu Würmern.

Ein lokalisiertes Giemen und Pfeifen gibt es bei Asthma oder COPD nicht. Typisch ist dort der ubiquitäre Befund. Wiederholt lokalisierte Auskultationsbefunde müssen deshalb endoskopisch abgeklärt werden, mahnte Dr. Jens Schreiber, Magdeburg, beim Pneumologenkongress in
Hannover. Ein exspiratorisches Giemen über dem rechten Unterlappen kann beispielsweise ein Polyp oder ein Fibroadenom sein oder auch eine lokale Stenose auf Höhe der Trachea und des Larynx.

Zeigen sich in der Inspiration heftige Stridorgeräusche mit Dyspnoe, könnte es sich auch um eine „vocal cord dysfunction" (VCD) handeln. Die tritt bei Asthma häufiger auf als bei Lungengesunden. Beobachtet man im Anfall einen Stimmritzenschluss, so ist das pathologisch, betonte Schreiber.
Auch Reisemitbringsel können asthmaähnliche Symptome hervorrufen. Eine tropische pulmonale Eosinophilie verursachen die endemisch in Südostasien und afrikanischen Ländern südlich der Sahara vorkommenden Filarien. Die Larven werden durch Moskitos auf den Menschen übertragen, siedeln sich in Lymphgefäßen an und setzen Mikrofilarien in die Blutbahn frei. Die daraufhin entstehende Immunreaktion führt $\mathrm{zu}$ asthmaähnlichen Symptomen und später zur Lungenfibrose. Auf eine Asthmatherapie spricht eine solche Infektion nicht an. Bei einer entsprechenden Reise in der Anamnese sollte man ggf. ein Tropeninstitut einschalten. 